\title{
Review of Decentralized Finance Applications and Their Total Value Locked
}

\author{
Viktorija Stepanova, Ingars Eriņš \\ Riga Technical University, Kalku street 1, Riga, Latvia
}

\begin{abstract}
Currently, in the world there is a growing interest in the digital economy including the blockchain technology. Decentralized Finance (DeFi) is one of the leading current blockchain technology-related trends. The paper aims to provide an overview of the opportunities, advantages and shortcomings of this technology, as well as to summarize information on 12 most popular DeFi applications, using Total Value Locked indicators for the period of 34 months.
\end{abstract}

Keywords - Blockchain, Decentralized Finance, Decentralized Applications, Total Value Locked.

\section{Introduction}

The publication by Satoshi Nakamoto "Bitcoin: A Peer-to-Peer Electronic Cash System" [1] sparked a growing global interest in the blockchain technology since 2008. The technology was conceived as a tool to solve double-spending problems and diminish the role of intermediaries in financial transactions. Blockchain allows recording every transaction performed in the network in a distributed ledger, ensuring the transaction may not be altered or deleted. Network users, in their turn, may connect to the system in real time and check the content of each transaction.

Thus, there is no longer need to rely on intermediaries and pay for their services, since the technology has replaced intermediaries who used to

DOI: $10.18421 /$ TEM101-41

https://doi.org/10.18421/TEM101-41

Corresponding author: Viktorija Stepanova,

Riga Technical University, Kalku street 1, Riga, Latvia.

Email: viktorija.stepanova@rtu.Iv

Received: 22 December 2020.

Revised: 04 February 2021.

Accepted: 12 February 2021.

Published: 27 February 2021.

(c) BY-NC-ND (C) 2021 Viktorija Stepanova \& Ingars Eriňš; published by UIKTEN. This work is licensed under the Creative Commons Attribution-NonCommercial-NoDerivs 4.0 License.

The article is published with Open Access at www.temjournal.com supervise the system. In 2009, the first decentralized digital currency Bitcoin was developed as a continuation of this idea; nowadays it is one of the most popular cryptocurrencies in the world. The main characteristic of cryptocurrency is that it does not belong to any centralized register, definite bank or economy of some country. In contrast to the traditional payment systems, blockchain is maintained by the network users themselves. As a result, bitcoin has not only solved the doublespending problem, but also created the concept of a digital asset, as until then all digital used to be easily copiable. According to CoinMarketCap [2] data, as of 30 October 2020, there were 7,560 cryptocurrencies with the total market capitalization amounting to $\$ 390,118,177,992$.

Since bitcoin was launched on the market, the community of developers and researchers has been studying the challenges and opportunities offered by the new technology. Blockchain remains a topical issue globally; the number of related publications keeps growing exponentially. Vitalik Buterin was one of the first crypto currency and blockchain enthusiasts of that time. In 2013, he published his white paper "A Next Generation Smart Contract \& Decentralized Application Platform" [3], where he presented the idea of an integrated blockchain that would allow any person to develop their own applications in a single environment. Initially, Ethereum protocol was conceived as an improved version of cryptocurrency, but the result surpassed financial protocols in a sense that it allows developing unique projects that are not essentially related to money and finance. In January 2014, the establishment of Ethereum platform was officially announced.

In the third quarter of 2020, Decentralized Finance was recognized the leading current trend since the ICO madness, which had been observed all over the world at the end of 2017 and beginning of 2018. In the report by ConsenSys Codefi [4], it was pointed out that it may be explained by the improved user experience offered by DeFi applications - nowadays private individuals take more interest in the opportunity to earn money from the fluctuations of the cryptocurrency exchange rates, to invest money gaining higher interest than at the bank, rather than gain additional income using traditional ways that for 
many years have been offered by the financial market.

The paper aims to provide an overview of the Decentralized Finance and to analyze the Total Value Locked of the DeFi applications.

Metadata analysis is used in the paper in order to summarize research results of numerous papers and answer the research questions.

The following research questions have been set in this paper:

RQ1: What are the main general principles of DeFi and what opportunities it offers?

RQ2: What are the most popular DeFi applications and what Total Value Locked is?

RQ3: What advantages and gains are offered by DeFi solutions?

\section{Decentralized Finance}

Traditional financial services are fully centralized. Centralized Finance (CeFi) suggests that asset owners and traders rely on some centralized institution in order to be eligible to manage their funds and make transactions with one another. Centralization of finance allows entrusting important decisions and communication to field experts. $\mathrm{CeFi}$ enables users to make fair transactions and evade improper financial management, fraud and corruption.

Thanks to the blockchain technology and Ethereum, market players gained opportunity to diminish the role of intermediaries, transferring the existing financial services to the blockchain and cryptocurrency environment. Ethereum is a blockchain-powered open software platform that gives developers opportunity to create and publish decentralized applications. The platform allows writing a code or the so-called smart contract that runs a financial service and automates contract performance between two parties excluding the third party. Smart contact ensures high accuracy, transparency, speed and security, since the information on fulfillment of contract requirements is available to all stakeholders. DeFi is a set of decentralized applications (dApps) that automate financial services based on the blockchain technology without any centralized control. The majority of DeFi applications are developed in Ethereum environment using an open code, which enables the developers to create new protocols on the basis of the existing ones and combine them among themselves. The users may also join and combine several financial services, which open wider opportunities for making transactions in the network. DeFi is sometimes called "Money Lego" because it offers unlimited combination opportunities. DeFi is rapidly becoming the main trend in Ethereum development and, according to DeFi Pulse data [5], as of 29 October 2020, dApp users entrusted more than 11 billon USD in cryptocurrency to smart contracts. DeFi networks allow cryptocurrency owners to lend or borrow their assets to other network users, recording their value in the protocol. John Wagster, a lawyer from a law firm Frost Brown Todd, at the webinar "Understanding Blockchainbased Decentralized Finance" [6] pointed out that DeFi ecosystem provides opportunity to lenders and investors to earn a lot more than offered by $\mathrm{CeFi}$ market.

DeFi includes 3-D (Digitalization, Decentralization, and Democratization) concepts of sustainable development that motivate the users to employ the blockchain technology in financial services:

- Digitalization - the use of digital technologies that influences all economic spheres, everyday life, work and communication;

- Decentralization - removal of control from large banks / financial institutions and division of power among wider public, which reduces costs of financial transactions and allows all participants to benefits from network effects and widen their opportunities;

- Democratization - equal opportunities for all, making financial services accessible to anyone irrespective of the social status or geographic location. A mobile phone and access to the Internet are everything one needs to access $\mathrm{DeFi}$;

DeFi ecosystem is characterized by a set of characteristics; it comprises the following main elements:

Digital asset - DeFi ensures full control of personal assets. The ability to digitally represent any item belonging to an individual and to ascribe value to it creates new market opportunities in the world of finance. Digital assets include:

- Cryptoasset - intangible personal property that serves as a means of exchange in the decentralized electronic environment and that does not have a tangible asset backing in, e.g., the form of gold;

- Stablecoin - a cryptoasset characterized by stability of its value, which guards its owner from price volatility. The value of a stablecoin is algorithmically added to the value of another real asset (for example, market price of gold or fiat money);

- Global stablecoins - digital assets with characteristics of stable cryptocoins that are created by large enterprises with a large user base (for example, Facebook "money" - Libra [7]).

Open-source software - software code is freely available to everyone, which ensures higher credibility to the platform. Anyone can analyze the code and check whether any malicious coding is 
hidden at the background. Moreover, this code may be used for personal needs, for example, modifying and improving existing solutions or developing new ones;

Coequal (P2P) market - the environment where all market participants have equal rights. In the market of that kind, all participants are able to communicate among themselves directly without intermediaries;

Smart contracts - a computer protocol that ensures conclusion of contracts and their automatic execution between two parties. The smart contract stipulates conditions and acts as a digital execution agent, which provides a programmed opportunity to control any financial transaction in the network without intermediaries.

Decentralized payments and transfers - the basis of DeFi ecosystem that helps create a more open economic system for the people irrespective of their social status, geographic location or political system therein and helps large financial institutions better serve wholesale and retail customers.

Decentralized loans and credits - one of the most popular ways to gain passive income. Anyone has an opportunity to use a personal digital asset as collateral for receiving a loan and/or invest in the credit resources within the selected protocol to gain profit. The majority of lending protocols offer floating interest rates that are automatically adjusted depending on supply and demand for a definite asset. There is only one intermediary between the lender and the borrower: blockchain protocol that allows participants to interact directly - the earned interest is received automatically. In addition, decentralized lending remains anonymous. There is no need for checking the credit score, which facilitates the process of raising finance.

Decentralized exchanges (DEX) - coequal opens trading platforms that transplant the basic functions of the traditional stock exchanges in the decentralized environment, replacing the central websites with protocols. Platforms allow traders to buy, sell or exchange one digital asset for a different asset available on the exchange without the need to input and output funds to third parties, which reduces transaction costs and increases process speed.

Derivative - a derived financial instrument that guards the buyer from future price fluctuations. The buyer signs a contract on the purchase of an asset for a fixed price on a definite date in future. These protocols enable two parties to exchange one asset for another. Different derivatives let traders gain profit from the fluctuations in the price of cryptoassets, buying them for lower and selling them for a higher price.

Asset management - an asset management process aimed at gaining investment income. Asset management protocols give investors opportunity to entrust their funds to a smart contract or fund manager. Transparency of DeFi allows users to monitor how their assets are managed and better understand where the related costs come from.

\section{DeFi Total Value Locked}

There are many different DeFi projects; many of them are developing rapidly. The amount of invested funds that may be expressed in monetary terms is analyzed within the paper in order to stress the topicality of the reviewed theme and to organize upto-date information on the most popular DeFi platforms.

The data of DeFi Pulse portal were used in order to examine the volume of funds invested in every DeFi platform. DeFi Pulse is one of the tools that help users to make informed investment decisions. Every day DeFi Pulse publishes information on the total value of the platforms - Total Value Locked (TVL), calculating the existing protocol Ether (ETH) and ERC-20 balance and multiplying it with the price in USD. According to DeFi Pulse data, the Total Value Locked in the dApp on 29 October 2020 was 11,288 billion USD and kept growing (Figure 1).

All | 1 Year | 90 Day | 30 Day

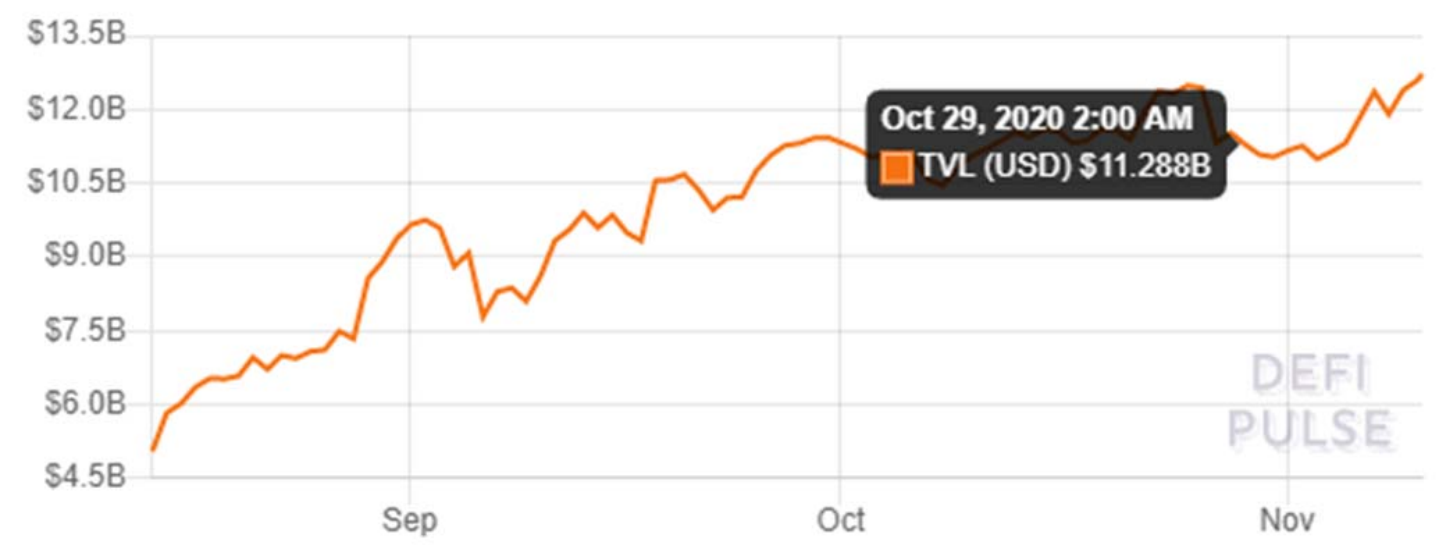

Figure 1. Total Value Locked in the decentralized financial applications [5] 
Examining the most popular DeFi platforms, the data on the TVL in 12 most popular dApp as of 29 October, 2020, were considered. The list of the analyzed platforms is given below:

- Payment and transfer platforms: Flexa, Lightning Network, xDai;

- Lending platforms: Maker, Compound, Aave;

- DEX platforms: Uniswap, Curve Finance, Balancer;

- Derivatives platforms: Synthetix, Nexus Mutual, Erasure;

- Asset management platforms: WBTC, Harvest Finance, yearn finance;

The following DeFi Pulse data were used to visualize the development of DeFi projects (Figure 2):
- Total Value Locked (TVL) in each platform on the launch date of the solution in order to appreciate the start-up capital of the platform;

- TVL in each platform on 29 October, 2020, in order to obtain the updated information on the funds invested in the platform and the total TVL growth;

- TVL in each platform on 29 October, 2019, in order to gain clear understanding of the progress made by each platform in one-year time;

- TVL of each platform is given as of 1 September, 2020, when the newest of the 12 analyzed platforms was established. This allows considering the progress reached in the period of two months;

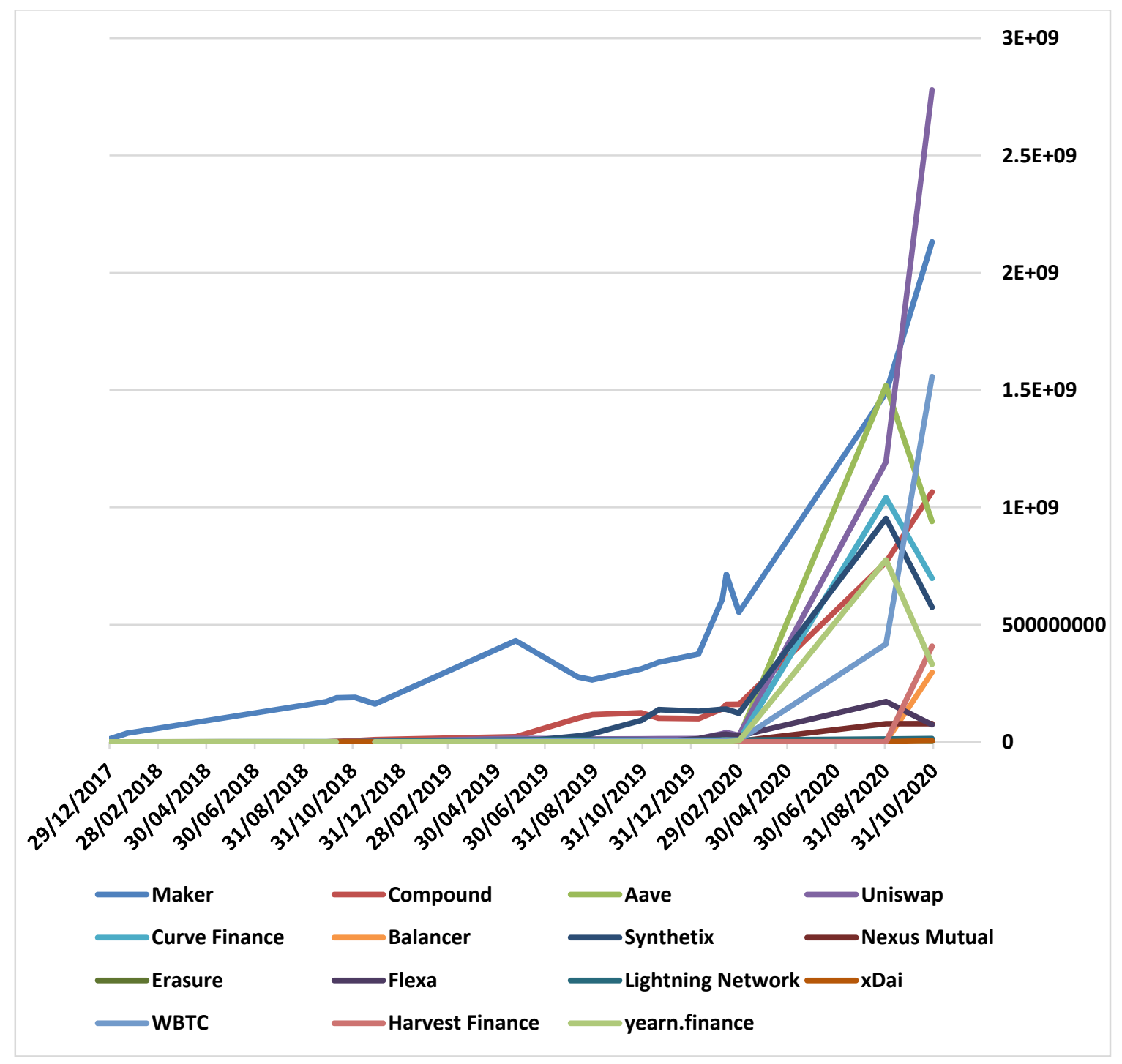

Figure 2. Development of DeFi projects

The newest data (29 October, 2020) demonstrate that the largest user funds are invested in Uniswap (TVL $=2,780,000,000 \quad$ USD). Uniswap (https://uniswap.org/) has introduced the automated liquidity protocol that enables users to quickly exchange ERC20 tokens. Anyone can lend their cryptocurrency to special reserves called liquidity pools, creating liquidity portfolio in order to gain profit. Uniswap uses a model that is called an automated market maker (AMM) instead of the order 
book. Currently, liquidity providers receive a transaction fee of $0.3 \%$ for each transaction. Uniswap protocol was created in 2018 with the initial TVL of 35,858 USD.

Maker (TVL $=2,132,000,000$ USD) is the second most popular DeFi solution. Maker (https://makerdao.com/en/) is the oldest of the considered platforms - Decentralized Autonomous Organization (DAO) was established in 2014. Introducing the original Dai (now Sai) stablecoin system, Maker published the Official Whitepaper in December 2017. Maker is a lending platform that supports stablecoins with the value attached to USD. Since 19 December, 2017, in almost three years' time, Maker's TVL has grown 1118.0 times (the initial TVL fixed in DeFi Pulse was 1, 907,000 USD).

WBTC - Wrapped Bitcoin asset management platform (TVL $=1,557,000,000$ USD) is the last in the top three DeFi leaders with the total value growth of $5,088,235.3$. The platform was launched on 28 November, 2018, with the initial value of only 306 USD and now it has been developing rapidly (the total annual value growth rate is 289.4). "Tokenized bitcoins" of the platform are in one-to-one correspondence with bitcoins. The idea of tokenization consists in transplanting the huge liquidity of bitcoin to Ethereum network. WBTC (https://wbtc.network/) is traded on different centralized and decentralized stock exchanges. The second version of the platform provides opportunity to tokenize any cryptoasset.

In the period under review, the lowest amount of funds was invested in three out of twelve considered platforms:

- Erasure protocol (https://erasure.world/) (TVL $=3,481,000$ USD) is based on a simple idea, "If you risk money on some information then it is probably good information so people will want to buy it from you. Skin in the game leads to better data". This derivatives platform allows uploading forecast flows with an aim to estimate which forecast has the highest value. Having purchased a forecast, the user receives a special key to new forecasts that give them opportunity to earn money. Erasure protocol was launched on 28 August, 2019, with initial TVL 1 USD. Today it is the platform with the largest annual value growth -452.6 . It is important to note that at the time this publication was written (16 November, 2020), Erasure lost its 3rd place among the top three derivatives platforms to an entirely new project HEGIC - the platform was launched with initial TVL of 1.43 billion USD on 11 October, 2020, and its TVL on 16 November, 2020, was fixed at 45.18 billion USD.
- xDai

(https://www.xdaichain.com/)

(TVL $=4,440,000$ USD) is a payment platform with extremely low commissions and stable currency value - USD 1:1 xDai, envisioned for daily transactions. The payments are made very quickly; the platform is user-friendly for the users with and without previous crypto experience. The platform was established on 10 October, 2018, with the initial TVL of 13 USD.

- Lightning Network (https://lightning.network/) is a payment platform that was established with an aim to solve bitcoin scaling problems, running integrated bidirectional payment channels between two cryptocurrency users. According to DeFi Pulse data, the users trust this platform for more than 2.5 years, having invested 14,110,000 USD in it. This platform demonstrates a stable, yet the lowest annual growth.

Harvest Finance (https://harvest.finance/)is the newest of the twelve most popular DeFi platforms; it is an asset management protocol that in essence ensures automated pooling and investment of the user funds in various strategies integrating other elements of DeFi ecosystem with an aim to generate higher profit (approximately $20-50 \%$ a year). In two weeks' time, its total value grew 1491.6 times from the initial value of 273,412 USD to $407,832,000$ USD.

Regarding the platforms that are younger than one year, the total two-month value growth rates demonstrate that Balancer (https://balancer.finance/) is another rapidly growing platform that permits making instant swop transactions on the web with modest commission fees. This platform is frequently compared with Uniswap. The total two-month value growth of Balancer amounted to 192.2. It should be noted though that at the time of writing of this paper (on 16 November, 2020), a new protocol SushiSwap vigorously pushed Balancer aside and joined the list of leaders among DEX platforms. An anonymous developer (Twitter user Chef Nomi) copied the Uniswap code in order to create SushiSwap (https://sushiswapclassic.org/), calling it a Uniswap 'evolution'. The only difference between Uniswap and SushiSwap is the introduction of SUSHI tokens that give opportunity to participate in voting regarding further development of the project and gain income from commissions. In accordance with this protocol, exchange commissions are $0.3 \%$, similar to Uniswap, but only $0.25 \%$ are channeled directly to the participants of the pool, the remaining $0.05 \%$ are transformed into SUSHI tokens and distributed among all token holders. This allows users to gain passive income even after signing out from the pool.

The results of analysis of 12 most popular DeFi platforms are summarized in Table 1. 
Table 1. Development of DeFi Platforms

\begin{tabular}{|c|c|c|c|c|c|}
\hline & $\begin{array}{c}\text { Name of the } \\
\text { platform }\end{array}$ & $\begin{array}{l}\text { Length of } \\
\text { service of the } \\
\text { platform } \\
\text { (full months) }\end{array}$ & $\begin{array}{c}\text { Total value } \\
\text { growth } \\
\text { (TVL as of } 29 \\
\text { October, } 2020 \\
\text { / to initial } \\
\text { TVL) }\end{array}$ & $\begin{array}{c}\text { Annual total } \\
\text { value growth } \\
\text { (TVL as of } 29 \\
\text { October, } 2020 \\
\text { / to TVL as of } \\
29 \text { October } \\
\text { 2019) }\end{array}$ & $\begin{array}{c}\text { Two-months } \\
\text { total value } \\
\text { growth } \\
\text { (TVL as of } 29 \\
\text { October, } 2020 \\
\text { / to TVL as of } \\
\text { 1 September, } \\
\text { 2020) }\end{array}$ \\
\hline \multirow{3}{*}{$\begin{array}{c}\text { Payment } \\
\text { and transfer } \\
\text { platforms }\end{array}$} & Flexa & 11 & 668.7 & - & 0.4 \\
\hline & $\begin{array}{c}\text { Lightning } \\
\text { Network }\end{array}$ & 33 & 991.6 & 1.8 & 1.2 \\
\hline & xDai & 24 & 341538.5 & 119.3 & 10.4 \\
\hline \multirow{3}{*}{$\begin{array}{l}\text { Loans and } \\
\text { credits } \\
\text { platforms }\end{array}$} & Maker & 34 & 1118.0 & 6.8 & 1.4 \\
\hline & Compound & 25 & 11435.3 & 8.6 & 1.4 \\
\hline & Aave & 9 & 2942.2 & - & 0.6 \\
\hline \multirow{3}{*}{$\begin{array}{c}\text { DEX } \\
\text { platforms }\end{array}$} & Uniswap & 23 & 77528.0 & 219.6 & 2.3 \\
\hline & $\begin{array}{c}\text { Curve } \\
\text { Finance }\end{array}$ & 8 & 801.6 & - & 0.7 \\
\hline & Balancer & 8 & 2340157.5 & - & 192.2 \\
\hline \multirow{3}{*}{$\begin{array}{c}\text { Derivatives } \\
\text { platforms }\end{array}$} & Synthetix & 14 & 21.9 & 6.2 & 0.6 \\
\hline & $\begin{array}{l}\text { Nexus } \\
\text { Mutual }\end{array}$ & 17 & 3261083.3 & 41.9 & 1.0 \\
\hline & Erasure & 14 & 3481000.0 & 452.6 & 0.5 \\
\hline \multirow{3}{*}{$\begin{array}{c}\text { Asset } \\
\text { management } \\
\text { platforms }\end{array}$} & WBTC & 23 & 5088235.3 & 284.9 & 3.7 \\
\hline & $\begin{array}{l}\text { Harvest } \\
\text { Finance }\end{array}$ & 1 & 1491.6 & - & 1491.6 \\
\hline & yearn.finance & 8 & 6106.1 & - & 0.4 \\
\hline
\end{tabular}

\section{Advantages and Disadvantages of DeFi}

Undoubtedly, rapid development of $\mathrm{DeFi}$ ecosystem proves that application of new technologies offers many advantages to market participants as compared to the use of traditional financial services. On the other hand, there are some associated limitations and risks. Benefits and limitations related to DeFi services are presented in Table 2 .

Table 2. Advantages and Disadvantages of DeFi

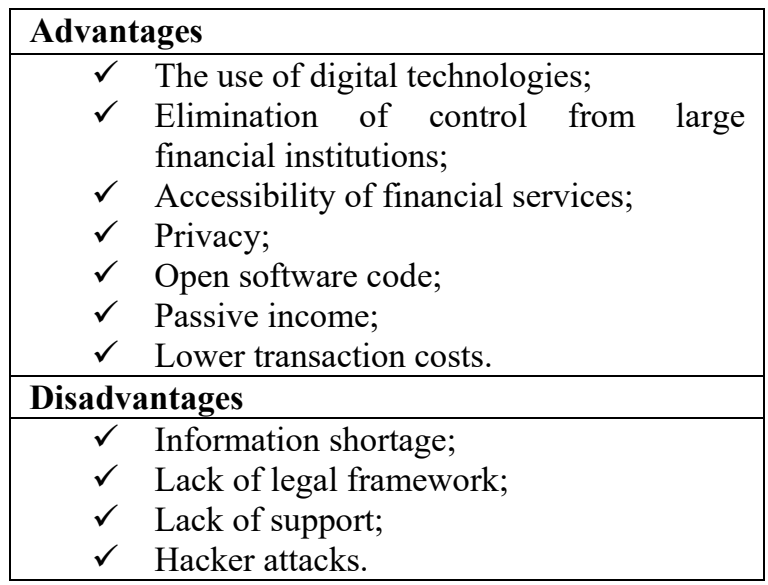

Exclusion of the financial intermediary is one of the main advantages; it helps reduce costs of financial transactions and allows users to follow financial flows themselves, as well as to interact among themselves. It is also important that DeFi solutions facilitate the trading process all over the world, providing opportunity to make transactions more efficiently and quickly. DeFi opens wider opportunities for the users, who are willing to invest their assets and quickly increase their income, than the traditional financial market. In spite of definite advantages, it is important to evaluate the associated risks and limitations that hinder DeFi deployment by the general public. One of the limitations is that only comparatively few people have heard something about opportunities offered by DeFi applications and yet smaller number use them on a daily basis. Information is available only in some internet sources; it is mainly intended for the people with preliminary knowledge and skills in IT/finance/crypto area. Undeniably, many books and scientific publications have been dedicated to DeFi solutions, they may help people develop deeper understanding of the related issues (e.g., [8], [9], [10]), but so far it is not sufficient to put DeFi solutions to a wider use. It may also be mentioned that the number of blockchain developers in the world is limited, and those who for some time work with this technology are rather experimenters, which does not fully exclude the possibility that mistakes may occur in the process of dApp development. The lack of legal framework and standards is another 
limitation frequently mentioned by DeFi users and developers. In order to regulate DeFi services, the following aspects will have to be considered in every country separately and internationally: international financial regulations, general issues in the area of tax legislation, data protection principles and many other regulatory enactments, rights and legal aspects that may be associated with provision of decentralized financial services. In the case of $\mathrm{DeFi}$, laws are replaced by a code that is normally developed by programmers not involving a wide range of regulators. For this reason, in the case of confusion associated with transactions or any other problems, it is difficult to receive any support from official state representatives. Hacker attacks are one of the risks that may affect DeFi users. The more user funds are invested on decentralized platforms, the more malicious users are attracted to the same platforms. The attackers regularly hack DeFi networks and steal user funds. Therefore, it is important to assess all gains and risks before investing money in some platform.

\section{Conclusion}

In the framework of this paper, the information about decentralized finance has been summarized and organized; the overview of the most popular DeFi applications has been provided. Total value indicators of 12 platforms for the period of 34 months were summarized using DeFi Pulse tool. As a result of data analysis, it has been concluded that at the beginning of October, 2020, the largest amount of funds was invested in Uniswap cryptocurrency exchange. Two most rapidly developing asset management platforms are WBTC, the platform that users trust for two years, and an entirely new platform Harvest Finance that was launched in the DeFi market two months ago. The loans and credit platform Maker in the oldest running platform in the DeFi market, it takes the second place in the ranking in terms of invested funds after Uniswap.

In the paper, the main principles, advantages and shortcomings of DeFi have been determined, which may help raise reader awareness of relatively new opportunities available in the financial market and motivate stakeholders to, for example, address the issues emerging in the area of legal framework and support provision. Despite definite risks and shortages, data analysis results attest that the amount of invested funds and the number of DeFi users have been growing rapidly and that may motivate developers and research community to conduct research and make new discoveries.

\section{References}

[1]. Nakamoto, S. (2020). A peer-to-peer electronic cash system.. Bitcoin. Retrieved from: https://bitcoin.org/bitcoin.pdf [accessed: 10 December 2020].

[2]. CoinMarketCap. (2018).Global Charts. Total Market Capitalization. Retrieved from:

https:/coinmarketcap.com/charts/ [accessed: 30 October 2020].

[3]. Buterin, V. (2014). A next-generation smart contract and decentralized application platform. white paper, 3(37). Retrieved from: https://github.com/ethereum/wiki/wiki/White-Paper [accessed: 10 September 2020].

[4]. Muzzy Everett, J. B. (2020). DeFi Report. An analysis of Ethereum's decentralized finance ecosystem in q3 2020 (No. 3). ConsenSys Codefi.

https://f.hubspotusercontent10.net/hubfs/4795067/Cod efi/consensys-q3-defi-report.pdf [accessed: 29 October 2020].

[5]. Data analytics site. (2020). DeFi Pulse. Retrieved from: https://defipulse.com/ [accessed: 29 October 2020].

[6]. Wagster, J. (2020). Understanding Blockchain-based Decentralized Finance [Online webinar]. [Participated: 27 October 2020].

[7]. Ebbage, A. (2019). New crypto on the block: There's a new cryptocurrency in town, but the long-rumoured stablecoin Libra will have to overcome some major obstacles to become an investible asset. Engineering \& Technology, 14(10), 50-53.

[8]. Popescu, A. D. (2020). Decentralized Finance (DeFi)The Lego of Finance. Social Sciences and Education Research Review, 7(1), 321-348.

[9]. Darren, L., Daryl, L., Sze, J.T., Kho, K. , Azmi Erina, Lee TM, O. B. (2020). How to Defi?, CoinGecko.

[10]. Kumar, M., Nikhil, N., \& Singh, R. (2020, June). Decentralising Finance using Decentralised Blockchain Oracles. In 2020 International Conference for Emerging Technology (INCET) (pp. 1-4). IEEE. 